\title{
Additively manufactured small-diameter vascular grafts with improved tissue healing using a novel SNAP impregnation method
}

\author{
Fatemeh Kabirian ${ }^{1,2,3}$ (D) | Peiman Brouki Milan² | Ali Zamanian | Ruth Heying ${ }^{3}$ | \\ Masoud Mozafari ${ }^{2}$
}

${ }^{1}$ Bioengineering Research Group, Nanotechnology and Advanced Materials Department, Materials and Energy Research Center (MERC), Tehran, Iran

${ }^{2}$ Department of Tissue Engineering and Regenerative Medicine, Faculty of Advanced Technologies in Medicine, Iran University of Medical Sciences, Tehran, Iran

${ }^{3}$ Cardiovascular Developmental Biology, Department of Cardiovascular Sciences, KU Leuven, Leuven, Belgium

\section{Correspondence}

Masoud Mozafari, PhD; Department of Tissue Engineering and Regenerative Medicine, Faculty of Advanced Technologies in Medicine, Iran University of Medical Sciences, Tehran, Iran.

Email: mozafari.masoud@gmail.com

\begin{abstract}
The vascular network has a complex architecture such as branches, curvatures, and bifurcations which is even more complicated in view of individual patients' defect anatomy requiring custom-specifically designed vascular implants. In this work, 3D printing is used to overcome these challenges and a new shorter impregnation method was developed to incorporate S-nitroso- $\mathrm{N}$-acetyl-D-penicillamine (SNAP) as a nitric oxide (NO) donor to printed grafts. The 3D-printed small-diameter vascular grafts (SDVGs) were impregnated with SNAP solution during SNAP synthesis (S1) or with SNAP dissolved in methanol (S2). The advantage of the newly developed S1 impregnation method is the elimination of the synthesis step by direct impregnation inside the S1 solution. Scanning electron microscopy imaging reveals the successful crystal formation in both methods. The results demonstrate that both S1- and S2-impregnated grafts, after covering with polycaprolactone topcoat, can release $\mathrm{NO}$ in a controlled manner and in the physiological range $\left(0.5-4.0 \times 10^{-10} \mathrm{~mol} \mathrm{~cm}^{-2} \mathrm{~min}^{-1}\right)$ over a 15 days period. The created grafts with a NO-releasing surface have also shown bactericidal effect while the healing properties of the implant were improved by promoting migration and proliferation of endothelial cells (ECs). These results suggest that incorporation of 3D printing technology with the newly developed S1 impregnation of SNAP can optimize and shorten the manufacturing process of the next generation of patient-based antibacterial SDVGs with a higher attraction for ECs.
\end{abstract}

\section{KEYWORDS}

3D printing, additive manufacturing, nitric oxide release, small-diameter vascular grafts, SNAP impregnation

\section{1 | INTRODUCTION}

The growing prevalence of cardiovascular diseases is leading to an increase in required vascular surgeries. One of the main vascular replacement procedures is bypassing the obstructed vessel with an autologous blood vessel. The application of autologous grafts is limited in terms of accessibility, size, mechanical properties, and harvesting issues which makes the synthetic grafts an appropriate candidate (Pashneh-Tala, MacNeil, \& Claeyssens, 2015).

Despite the application of sterile surgical techniques, bacterial contamination is considered as a main clinical complication of current synthetic vascular grafts, especially for small-diameter vascular grafts (SDVGs, <6 mm) which can cause graft failure (Kabirian, Amoabediny, Haghighipour, Salehi-Nik, \& Zandieh-Doulabi, 2015; Kabirian, Ditkowski, 
Zamanian, Heying, \& Mozafari, 2018; Pashneh-Tala et al., 2015; Radakovic et al., 2017; Rao et al., 2018). Bacteria start to colonize immediately after implantation especially during the critical first $6 \mathrm{hr}$ which is also known as the "decisive period." Since the management of this period is directly related to success or failure of implantation and the host's defenses are not able to completely combat the infection process during this period (Zilberman \& Elsner, 2008), antimicrobial agents are used in the form of systemic or controlled release systems. Common approaches are the controlled release of antibiotic or silver nanoparticles which can result in the development of antibiotic resistance of bacteria or cytotoxicity, respectively (Vermote \& Van Calenbergh, 2017; Wo et al., 2017). A commonly used strategy is to cover the surface of biomaterials with endothelial cells (ECs) which create a surface with antifouling properties. Although the endothelium can solve such problems, cell culture is a time-consuming and expensive method with restricted "offthe-shelf" availability which can limit the clinical success.

Nitric oxide (NO) is a vital endogenous molecule which can overcome these challenges without resulting in undesirable adverse effects such as antibiotic resistance, cytotoxicity, and expensiveness (Wo et al., 2017). By engineering the blood-contacting surfaces to release NO in a controlled manner, it can be possible to mimic the properties of the endothelium without its clinical limitations. Controlled release of NO from medical devices can also improve the tissue regeneration of implants by promoting ECs to migrate from adjacent tissues (Gao et al., 2017; Yang et al., 2015) which in turn can increase the success rate of graft implantation.

Various molecules such as S-nitroso- $\mathrm{N}$-acetyl-D-penicillamine (SNAP) with the ability to release $\mathrm{NO}$ are appropriate candidates to be used as NO donor. SNAP is a biocompatible and most stable donor which is derived from penicillamine, an FDA approved agent (Carpenter \& Schoenfisch, 2012; Colletta et al., 2015; Riccio \& Schoenfisch, 2012). One of the simplest procedures to load SNAP within the polymeric structures is the impregnation method. Recent studies have developed this approach for commercial medical devices by synthesis of SNAP and subsequent loading of synthesized SNAP by $24 \mathrm{hr}$ soaking in the dissolved donor solution (Brisbois et al., 2016; Colletta et al., 2015).

Although medical devices are commercially available to be impregnated with SNAP molecules, their fabrication based on patients' individual geometry of the pathologic site is advantageous. 3D printing is a layer by layer additive manufacturing process which can create grafts according to the designed 3D CAD model (Kabirian \& Mozafari, 2019). In the current study, fused deposition modeling (FDM) was used as a simple $3 \mathrm{D}$ printing method which creates the constructs by extruding and solidifying the polymer according to the CAD model. FDM is able to create objects from FDA-approved polymers like PLA without any chemical modifications which enhances the chance of the created model itself to become FDA approved (Touri, Kabirian, Saadati, Ramakrishna, \& Mozafari, 2019).

For the next generation of SDVGs, we offer a simple, fast, and patient-based method to fabricate SDVGs even in the hospital and before the operation. In this approach, the SDVG can be created according to imaging data obtained from a pathologic vessel which requires replacement. By converting the radiographic data to the CAD model, the SDVG can easily be printed and prepared for SNAP impregnation and implantation.

In this study, we combined the 3D printing technology with a novel modified SNAP impregnation method to prepare a NO-releasing controlled system. A new modified impregnation method has been developed, which can be performed during the SNAP synthesis process. Indeed, in the current study we aimed to simplify the process by combining the synthesis and impregnation steps and performing the impregnation step during the SNAP synthesis process. Therefore, the objective of this study is to improve and facilitate both fabrication and impregnation steps of antibacterial SDVGs with improved healing properties.

\section{2 | MATERIALS AND METHODS}

\section{1 | Materials}

Dimethyl sulfoxide (DMSO), thiazolyl blue tetrazolium, polycaprolactone (PCL, $M n=80,000$ ), N-acetyl-D-penicillamine (NAP), sodium nitrate, vanadium (III) chloride, sulfanilamide, and $\mathrm{N}$-(1-naphthyl) ethylene diamine dihydrochloride (NEDD) were purchased from Sigma-Aldrich (St. Louis, MO). Sulfuric acid $\left(\mathrm{H}_{2} \mathrm{SO}_{4}\right)$, ortho-phosphoric acid $\left(\mathrm{H}_{3} \mathrm{PO}_{4}\right)$, hydrochloric acid $(\mathrm{HCl})$, methanol, tetrahydrofuran (THF), Luria broth (LB), tryptic soy broth (TSB), and sodium nitrite $\left(\mathrm{NaNO}_{2}\right)$ were obtained from Merck. Calcium and magnesium free Dulbecco's phosphate buffered saline (PBS, pH 7.4), Dulbecco's modified Eagle's medium, fetal bovine serum (FBS), and penicillin/streptomycin were purchased from Invitrogen and Gibco, respectively. Human umbilical vein endothelial cells (HUVECs, NCBI Code: C554) were obtained from the Cell Bank of the Pasteur Institute of Iran.

\section{2 | Design and production of 3D-printed SDVGs}

The digital 3D-model of the graft was prepared in the Rhinoceros ${ }^{\circledR}$ CAD modeling package (version 5 SR8, Robert McNeel \& Associates). The dimensions of specimens were designed to be $10 \mathrm{~mm}$ in height and $4.5 \mathrm{~mm}$ in diameter with $0.2 \mathrm{~mm}$ wall thickness and saved in a stereolithography (STL) format. Using the CURA slicing software (Ultimaker BV, Geldermalsen, Netherlands), the STL file was sliced layer by layer and converted into a g-code. The g-code was imported to the homemade 3D printer which has been assembled in our lab according to a typical desktop FDM printer (Kabirian et al., 2018). The grafts were printed layer by layer via extruding PLA filaments through a $220^{\circ} \mathrm{C}$ nozzle with $200 \mu \mathrm{m}$ internal diameter. The PLA layers were deposited and solidified onto a $64^{\circ} \mathrm{C}$ platform while the infill density of the polymer was set at $100 \%$.

\section{3 | SNAP synthesis}

A modified version of the SNAP synthesis protocol was used in this study (Kabirian, Ditkowski, et al., 2019). Briefly, 1,000 mg of NAP was dissolved in $25 \mathrm{~mL}$ of methanol and consequently $15 \mathrm{~mL}$ of $1 \mathrm{M} \mathrm{HCl}$, $500 \mu \mathrm{L}$ of $\mathrm{H}_{2} \mathrm{SO}_{4}$ (98\%), and $724.5 \mathrm{mg}$ of $\mathrm{NaNO}_{2}$ were added and 
mixed, respectively. The solution was stirred for $15 \mathrm{~min}$ and ice-cooled for $45 \mathrm{~min}$ while the methanol was evaporated simultaneously under $\mathrm{N}_{2}$ flow. The dark-green crystals were rinsed and filtered with icecooled DI water and freeze-dried. The obtained crystals were stored in an amber vial at $-20^{\circ} \mathrm{C}$.

\section{4 | Preparation of SNAP-impregnated grafts}

According to the stoichiometry of the SNAP synthesis reaction (NAP $+\mathrm{HNO}_{2} \rightarrow$ SNAP; Chipinda \& Simoyi, 2006), $1 \mathrm{~mL}$ of the mother solution (which makes the SNAP) contains $28.43 \mathrm{mg}$ SNAP. Thus, $28.43 \mathrm{mg} \mathrm{ml}^{-1}$ SNAP was the selected concentration to prepare the swelling solution in parallel. Briefly, $1 \mathrm{~mL}$ of mother solution as Solution 1 (hereafter named S1) and $28.43 \mathrm{mg}$ SNAP dissolved in $1 \mathrm{~mL}$ of methanol as Solution 2 (hereafter named S2) were used to impregnate the grafts (Figure 1). Methanol was used as the solvent of SNAP for preparation of the $\mathrm{S} 2$ solution since it makes the main part of the S1 solution, the solubility of SNAP in methanol is maximum compared to other solvents (Wo et al., 2017), and because of its rapid evaporation. 3D-printed grafts were completely soaked in S1 or S2 solutions in darkness. After $24 \mathrm{hr}$, the grafts were rinsed three times in ice-cooled PBS and dried under $\mathrm{N}_{2}$ flow. To control the burst release and to prolong the NO flux, the impregnated grafts were dip-coated twice in the PCL solution prepared by dissolving $100 \mathrm{mg} \mathrm{PCL}$ in $1 \mathrm{~mL}$ of THF.

\section{5 | Graft inspection by scanning electron microscopy}

Pristine, S1-impregnated, and S2-impregnated grafts were sectioned longitudinally to observe their luminal surface. Specimens were prepared by locating on supporting stubs covered with carbon stickers and sputter coated with chromium. Secondary electron micrographs

(Figure 3b-i) were collected with SEM (Zeiss Sigma, Germany) at an accelerating voltage of $2 \mathrm{kV}$ under vacuum condition.

\section{6 | NO-release measurement}

To measure the NO-release at predetermined time points, the modified Griess assay was performed (García-Robledo, Corzo, \& Papaspyrou, 2014; Kabirian et al., 2015; Miranda, Espey, \& Wink, 2001). The grafts were soaked in $10 \mathrm{~mL}$ of PBS (pH 7.4) in darkness at $37^{\circ} \mathrm{C}$. At each time point, $150 \mu \mathrm{L}$ sample was collected for NO measurement and kept at $-20^{\circ} \mathrm{C}$. Since the half-life time of NO is approximately a few seconds, commonly its stable products including nitrite and nitrate were measured to determine the NO amount. Therefore, the nitrate content was reduced to nitrite and the nitrite amount was measured. Briefly, $50 \mu \mathrm{L}$ of the specimen and $50 \mu \mathrm{L}$ of $1: 1$ mixture of $2 \mathrm{~g}$ sulfanilamide in $100 \mathrm{~mL}$ of $\mathrm{DI}$ water and $3.44 \mathrm{~mL}$ of $\mathrm{H}_{3} \mathrm{PO}_{4}$ and $0.2 \mathrm{~g}$ NEDD in $100 \mathrm{~mL}$ of DI water were transferred in each well of a 96-well ELISA plate and gently mixed for 15 min in darkness. Next, $50 \mu \mathrm{L}$ of $\mathrm{VCl}_{3}$ solution, prepared by dissolving $400 \mathrm{mg} \mathrm{VCl} 3$ in $50 \mathrm{~mL}$ $1 \mathrm{M} \mathrm{HCl}$ was loaded to the well. The plate was incubated at $60^{\circ} \mathrm{C}$ for 60 min in darkness while covered with an adhesive film (VWR). Then the plate was cooled down to room temperature and the absorbance was measured at $550 \mathrm{~nm}$. The NO concentration was calculated using standard curves produced from serially diluted $\mathrm{NaNO}_{2}$ solutions.

\section{7 | Graft-bacterial interaction in solution}

To compare the antibacterial properties of the prepared grafts, the viability of bacteria was investigated in a solution culture. The inoculums of Gram-positive (Staphylococcus aureus, ATCC 8325-4) and Gram-negative (Escherichia coli, ATCC 25922) strains were prepared at a concentration of $10^{7} \mathrm{CFU} \mathrm{ml}^{-1}$ in PBS supplemented with $10 \%$

\section{S1 impregnation method}

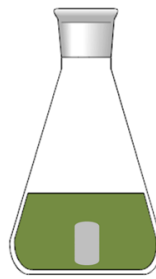

S1

(SNAP during synthesis)

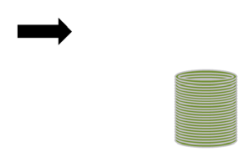

S1 impregnated 3d-printed graft
FIGURE 1 Schematic representation of $\mathrm{S} 1$ and S2 impregnation methods. Impregnation of 3D-printed grafts with (a) S1 method using SNAP solution during synthesis and (b) S2 method by dissolving synthesized SNAP in methanol. SNAP, S-nitroso- $N$-acetyl-D-penicillamine (b)

(a)

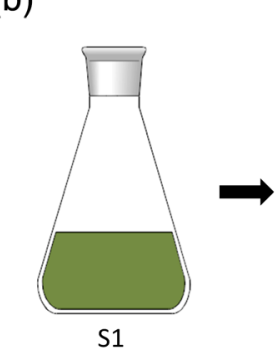

(SNAP during synthesis)
S2 impregnation method

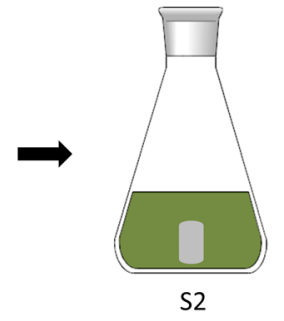

(SNAP dissolved in methanol)

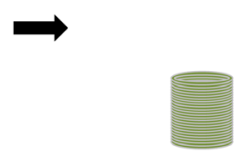

S2 impregnated 3d-printed graft 
TSB or LB, respectively. UV-sterilized S1, S2, and control grafts were immersed in $2 \mathrm{~mL}$ of bacterial solution for $24 \mathrm{hr}$ at $37^{\circ} \mathrm{C}$ with shaking at $200 \mathrm{rpm}$ in darkness. At time points of 6 and $24 \mathrm{hr}, 100 \mu \mathrm{L}$ sample from each tube were collected and serially diluted in sterile PBS for plate counting on TSB agar (S. aureus) or LB agar (E. coli). All experiments were repeated four times.

\subsection{Disk diffusion susceptibility test}

To evaluate the antibacterial potential of impregnated surfaces, the disk diffusion test was also carried out with Gram-positive (S. aureus, ATCC 8325-4) and Gram-negative (E. coli, ATCC 25922) bacteria. Disks with the same structure as SDVGs $(200 \mu \mathrm{m}$ thickness and $5 \mathrm{~mm}$ diameter) were created by the FDM printer, impregnated with the S1 and S2 method, and top coated with PCL. The solution culture of both strains was prepared at a density of $10^{5} \mathrm{CFU} \mathrm{ml} \mathrm{m}^{-1}$ and were homogeneously streaked on LB and TSB agar plates $(n=4)$, respectively (Lee et al., 2016; Pant et al., 2017; Thi, Lee, Le Thi, \& Park, 2018). Afterward, UV-sterilized disks were placed on inoculated agar plates and incubated at $37^{\circ} \mathrm{C}$. After $24 \mathrm{hr}$, the inhibition zones were observed and photographed with a camera.

\section{9 | Extract collection from fabricated grafts}

Ten milligrams of UV-sterilized S1, S2, and control grafts were soaked in $10 \mathrm{~mL}$ of DMEM medium at $37^{\circ} \mathrm{C}$ to obtain the extract. After $24 \mathrm{hr}$, the grafts were discarded from the extracts and kept in the fridge. Before the experiment, 10\% FBS and 1\% penicillin-streptomycin were added to the extracts to be prepared for cell culture (Pant, Goudie, Chaji, Johnson, \& Handa, 2018).

\subsection{0 | Cell culture}

Human umbilical vein endothelial cells were cultured in DMEM medium supplemented with $10 \%$ FBS and $1 \%$ penicillin-streptomycin in a humidified atmosphere containing $5 \% \mathrm{CO}_{2}$ at $37^{\circ} \mathrm{C}$.

\subsection{1 | Proliferation and morphology assessment}

To study the effect of the extracts on morphology and proliferation of ECs, a confluent monolayer of HUVECs were detached from T-75 flask and diluted in extracts. Afterward, $10^{4}$ cells/well were seeded in a 96-well plate $(n=3)$ and incubated at $37^{\circ} \mathrm{C}$. After $24 \mathrm{hr}$, cells were inspected by an inverted microscope (Olympus, Japan) and images were captured for further analysis.

\subsection{2 | Wound healing migration assay}

The effect of extracts on HUVEC migration was further investigated using the scratch assay. HUVECs at a concentration of $10^{4}$ cells/well were seeded in 96 -well plates $(n=3)$ and incubated at $37^{\circ} \mathrm{C}$. After obtaining a confluent monolayer, a vertical scratch was created with a $100-\mu l$ pipette tip across the center of the well, the culture media was aspirated and cells were rinsed twice with PBS to remove detached cells (Kabirian, Milan, Zamanian, Heying, \& Mozafari, 2019). The cellfree scratch wound is a model of graft borders after implantation where EC migration from the adjacent native vessel is crucial for endothelialization and graft integration. The wounded monolayers were incubated with extracts or fresh culture media (positive control) for $24 \mathrm{hr}$ at $37^{\circ} \mathrm{C}$. The inverted microscope (Olympus, Japan) was used to monitor the cell migration at 0 and $24 \mathrm{hr}$ after scarification by imaging. The obtained photographic images were further analyzed using the WimScratch online program.

\subsection{3 | Cytocompatibility assay}

To provide a proof of concept on the noncytotoxic potential of extracts, the MTT colorimetric assay was performed after 24 and $72 \mathrm{hr}$ of incubation with extracts. Briefly, HUVECs were suspended in extracts supplemented with $10 \% \mathrm{FBS}$ and $1 \%$ penicillin-streptomycin, and $5 \times 10^{3}$ cells/well were seeded in 96-well plates ( $n=6$ for each condition) and incubated at $37^{\circ} \mathrm{C}$ and $5 \% \mathrm{CO}_{2}$. The culture medium was replaced with fresh extracts at Day 2. At each time point, the medium was replaced with $110 \mu \mathrm{L}$ of $5 \mathrm{mg} / \mathrm{mL}$ 3-(4,5-dimethylthiazol-2-yl)-2,5-diphenyltetrazolium bromide in PBS and incubated for $4 \mathrm{hr}$ in $5 \% \mathrm{CO}_{2}$ atmosphere at $37^{\circ} \mathrm{C}$. Thereafter, the supernatant was aspirated and replaced with $110 \mu \mathrm{L}$ of DMSO and shaked for $15 \mathrm{~min}$ to dissolve the formazan crystals produced by viable cells. Hundred microliters of the supernatant was transferred into a new 96-well plate and the absorbance was measured at $570 \mathrm{~nm}$ by using a microplate reader (BioTek; Su et al., 2016; Zamani et al., 2017).

\subsection{4 | Statistical analysis}

All data were expressed as mean $\pm S D$. One- or two-way analysis of variance (ANOVA) was performed for statistical analysis and followed by Tukey or Bonferroni post-testing, respectively. A $P$ value of $<.05$ was considered significant.

\section{3 | RESULTS AND DISCUSSION}

\section{1 | Preparation of 3D-printed SDVGs}

The 3D printing technology used in the current study is a cost-effective, accurate, and fast approach with the ability to print patientspecific geometry. The SDVGs were successfully prepared from PLA filament using a FDM 3D printer according to a predesigned CAD model. Previously, we fabricated biodegradable 3D-printed SDVGs from PLA using the FDM machine (Kabirian et al., 2018; Kabirian, Ditkowski, et al., 2019; Kabirian, Milan, et al., 2019). In this study, to improve the healing and antibacterial properties of such SDVGs, a novel NO-releasing system was successfully incorporated.

\section{2 | SNAP impregnation of 3D-printed SDVGs}

A common method to incorporate SNAP into polymer matrices is the solvent swelling of SNAP (Brisbois et al., 2016; Colletta et al., 2015; 
Wo et al., 2017). In this procedure, the polymer matrix is immersed in a predetermined SNAP concentration in an appropriate solvent. The advantage of this method is the independence of loading the NO donor from the $3 \mathrm{D}$ printing process of the biomaterial. Therefore, this method is feasible for the preparation of commercially available devices or 3D-printed constructs.

In the current study, this typical and simple impregnation method was modified to an easier and shorter procedure to facilitate the preparation step. A novel impregnation technique has been used to create NO-releasing surfaces in which the grafts were soaked in the SNAP mother solution instead of synthesized SNAP being dissolved.

\section{3 | Inspection of 3D-printed and impregnated SDVGs with SEM}

$3 \mathrm{D}$ printing is an advantageous procedure to design and fabricate highly accurate and reproducible custom-based structures which can promisingly be used for cardiovascular tissue engineering applications
(Kabirian, Ditkowski, et al., 2019; Kabirian, Milan, et al., 2019). In the current study, SDVGs were successfully fabricated using the FDM 3D printing technology with PLA filaments (Figure 2a). The morphology of the printed SDVGs in form of pristine, S1 impregnated, and S2 impregnated were inspected by SEM and compared.

For pristine grafts, from the top view, Figure $2 \mathrm{~d}, \mathrm{~g}$ reveal the expected circular struts with sufficient attachment to the layer beneath and Figure 2b,c represent the smooth and homogeneous morphology of printed struts in the luminal view.

Figure 2e,f and Figure 2h,i indicate the crystals generated from S1 and S2 impregnation, respectively. The crystal structure of S2 crystals is in accordance with the crystal structure shown in the literature (Wo et al., 2015).

Comparing the two types of crystals, the crystals created from S1 are smaller in size, larger in population, and more uniformly distributed. Interestingly, the S1 crystals were dispersed more homogeneously and are in a broader range of sizes while the S2-impregnated crystals are more similar in size. This microscale roughness of PLA (a)

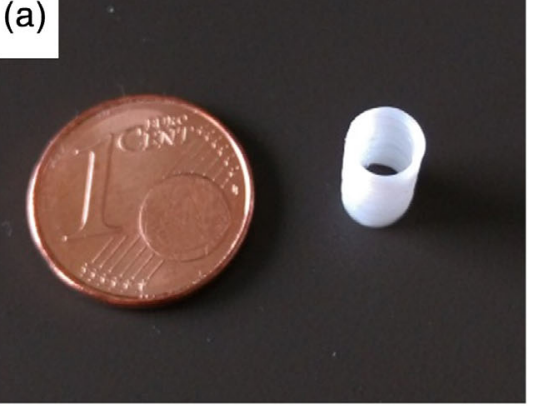

(d)
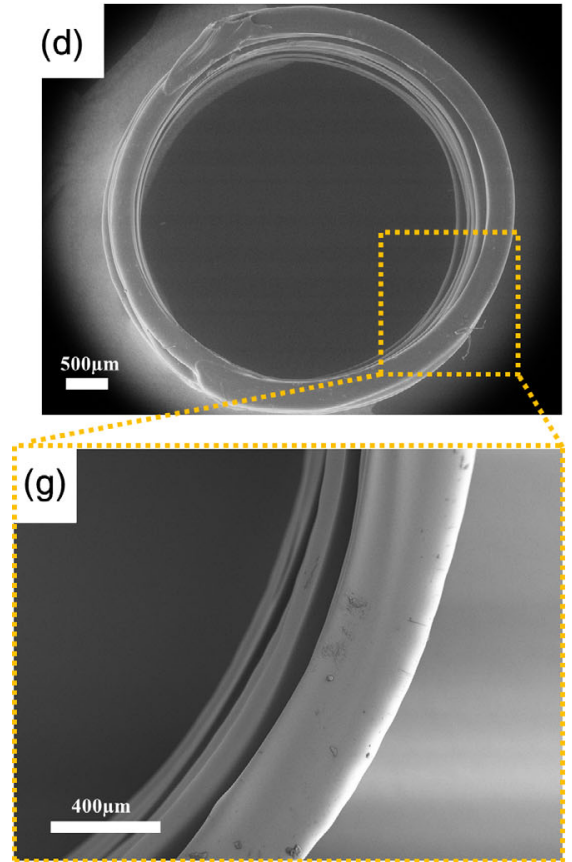

(b)
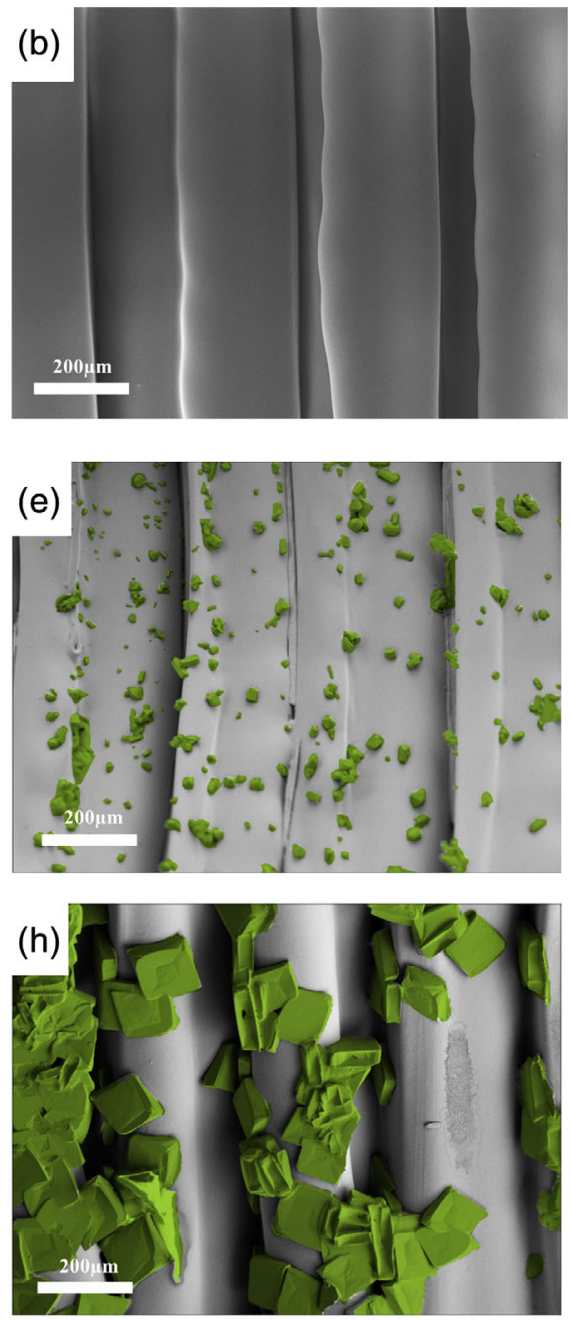
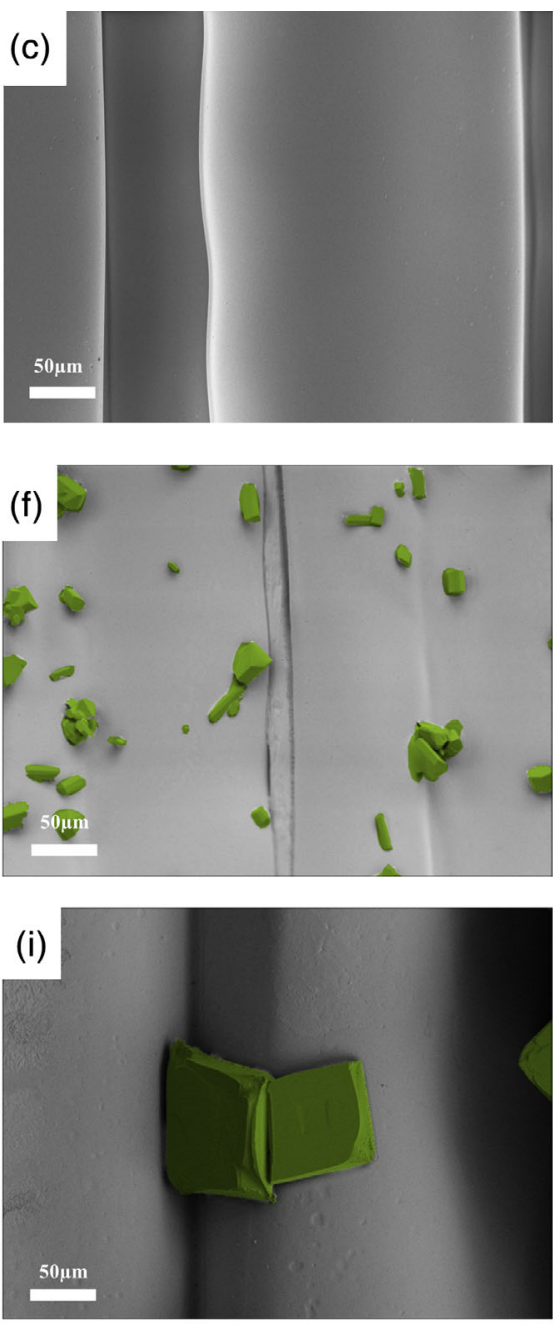

FIGURE 2 Photograph and SEM images of the fabricated vascular grafts with/without SNAP crystals. Macroscopic appearance of the 3D-printed graft (a). SEM images of the luminal surface of struts (b, c), cross-sectional view of 3D-printed grafts (d, g), luminal view of S1-impregnated struts (e, f), and S2-impregnated struts (h, i). Scale bars: (b, e, h) $200 \mu \mathrm{m}$, (c, f, i) $50 \mu \mathrm{m}$, (d) $500 \mu \mathrm{m}$, (g) $400 \mu \mathrm{m}$. SEM, scanning electron microscopy; SNAP, S-nitroso-N-acetyl-D-penicillamine 
after S2 impregnation is consistent with previous reports (Goudie, Brainard, Schmiedt, \& Handa, 2017).

A closer observation in higher magnifications (Figure 2f,i), demonstrated that S2 crystals were adsorbed at the external surface, while the S1 crystals grew from the inside of the struts and continued to the surface. This partially embedded morphology of S1 crystals into the SDVG matrix distinguishes it from S2 crystals that formed and covered the surface and each other. Therefore, it is reasonable that the S2 crystals covered a larger surface than S1 crystals.

\section{4 | NO measurement}

The release profile of NO from both S1- and S2-impregnated grafts are showed in Figure 3. The results are in line with the other reports of a primary burst release followed by a steady-state release in the physiological range (Wo et al., 2017). To control the initial burst release, the PCL double layers were top coated on the surface which remarkably decreased the release profile to the physiological range. On the first day of the experiment, a burst release of NO was observed due to the rapid dissolution of noncrystalized SNAP on the surface (Brisbois et al., 2016). This burst release is beneficial during the "decisive period" to prevent bacterial adhesion on the newly implanted surface when the host defenses cannot completely prevent the infection process (Zilberman \& Elsner, 2008). From the second day, the NO-release profile drops to the physiological range and fluctuated in this range. A plausible explanation for this finding may be that the SNAP molecules were in crystalline structure which takes time to be dissolved and resulted in long-term slow NO-release (Wo et al., 2017).

The results demonstrated that with the same concentration of SNAP, swelling time, and drying process, both impregnated grafts sustainably released NO which lasted for 15 days in the physiological range. There were no statistically significant differences in release kinetics between these two crystals and both crystals showed the same trend of release.

The partly penetrated morphology of S1 crystals into the grafts, which illustrate a possible firm attachment within SDVG bulk, can explain the same period of NO-release from this structure compared with S2 crystals while the crystals were smaller in size compared to
S2 crystals. It was reported in other studies that the long-term release of NO is related to crystal stability within the polymer matrix which is in agreement with the results of NO-release in the current study (Goudie, Brainard, et al., 2017).

Overall, we have introduced a novel shorter and easier method to impregnate the vascular biomaterials with a longer NO-release duration within the physiologic range.

\section{5 | In vitro antibacterial testing}

It has been shown that NO has antimicrobial properties because of its ability to damage the bacterial DNA, lipids, and proteins (Fang, 1997). The antibacterial effect of both S1 and S2 crystals were investigated under physiological conditions at $37^{\circ} \mathrm{C}$. Main pathogens associated with medical device infections, S. aureus as a Gram-positive and E. coli as a Gram-negative strains were investigated in this study.

First, the antibacterial effect of grafts was examined in liquid media. To exclude the effect of poor nutrition on the viability of bacteria, which may influence with the NO-release effect, bacteria were inoculated in PBS supplemented with $10 \%$ fresh growth media. The initial concentration of both strains were approximately $10^{7} \mathrm{CFU} \mathrm{ml}{ }^{-1}$, while after $6 \mathrm{hr}$ of incubation with the grafts the control microorganisms proliferated, the population of bacteria in presence of the NOreleasing grafts were reduced. After $24 \mathrm{hr}$ of incubation under shaking, the number of organisms incubated with control grafts reached the steady-state maximum level while the bacteria in presence of NOreleasing grafts were completely eradicated (Figure 4a,e).

Next, the inhibition of bacterial growth in the presence of grafts was studied on agar plates. The S1, S2, and control disks were placed on inoculated agar plates and the inhibition zones were inspected after $24 \mathrm{hr}$. While no inhibition zones were detected around control disks (Figure 4d,h), the inhibition diameter of S1 and S2 for S. aureus was $8 \mathrm{~mm}$ (Figure $4 \mathrm{~b}, \mathrm{c}$ ) and for E. coli $9 \mathrm{~mm}$ (Figure 4f,g). It can be concluded that NO molecules inhibit bacterial growth in vitro and therefore might be able to prohibit bacterial colonization and subsequent biofilm formation on the graft surface. This potential antibiofilm property is crucial for ECs to successfully cover the surface of the biomaterial in vivo.

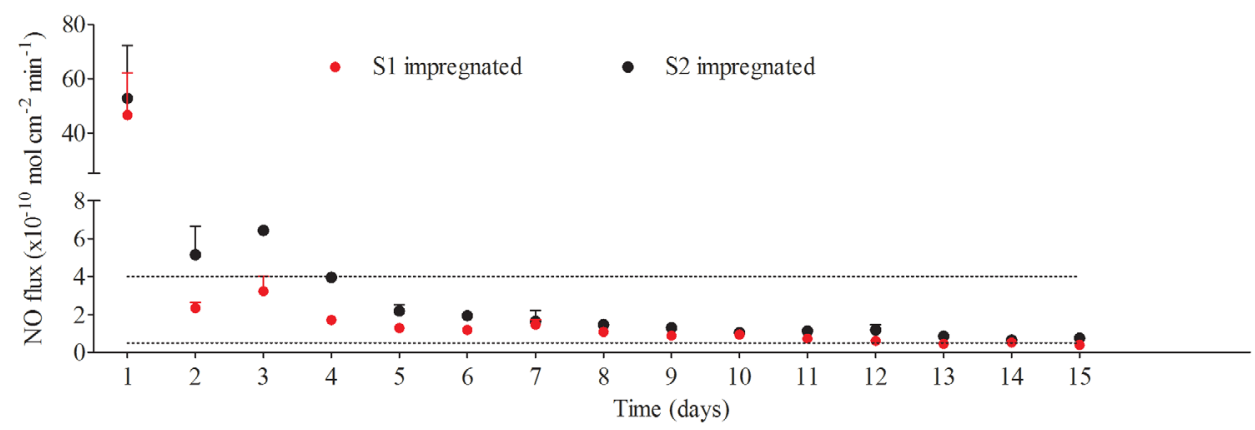

FIGURE 3 The in vitro NO-release profiles of S1- and S2-impregnated grafts. NO flux was measured in PBS at $37^{\circ} \mathrm{C}$ from both S1 and S2 crystals with PCL topcoat. The dotted lines indicate the physiological range of NO flux $\left(0.5-4.0 \times 10^{-10} \mathrm{~mol} \mathrm{~cm}^{-2} \mathrm{~min}^{-1}\right)$. All NO amounts were measured by a modified Griess assay. Data are expressed as mean $\pm S D(n=6)$. PBS, phosphate buffered saline; PCL, polycaprolactone 

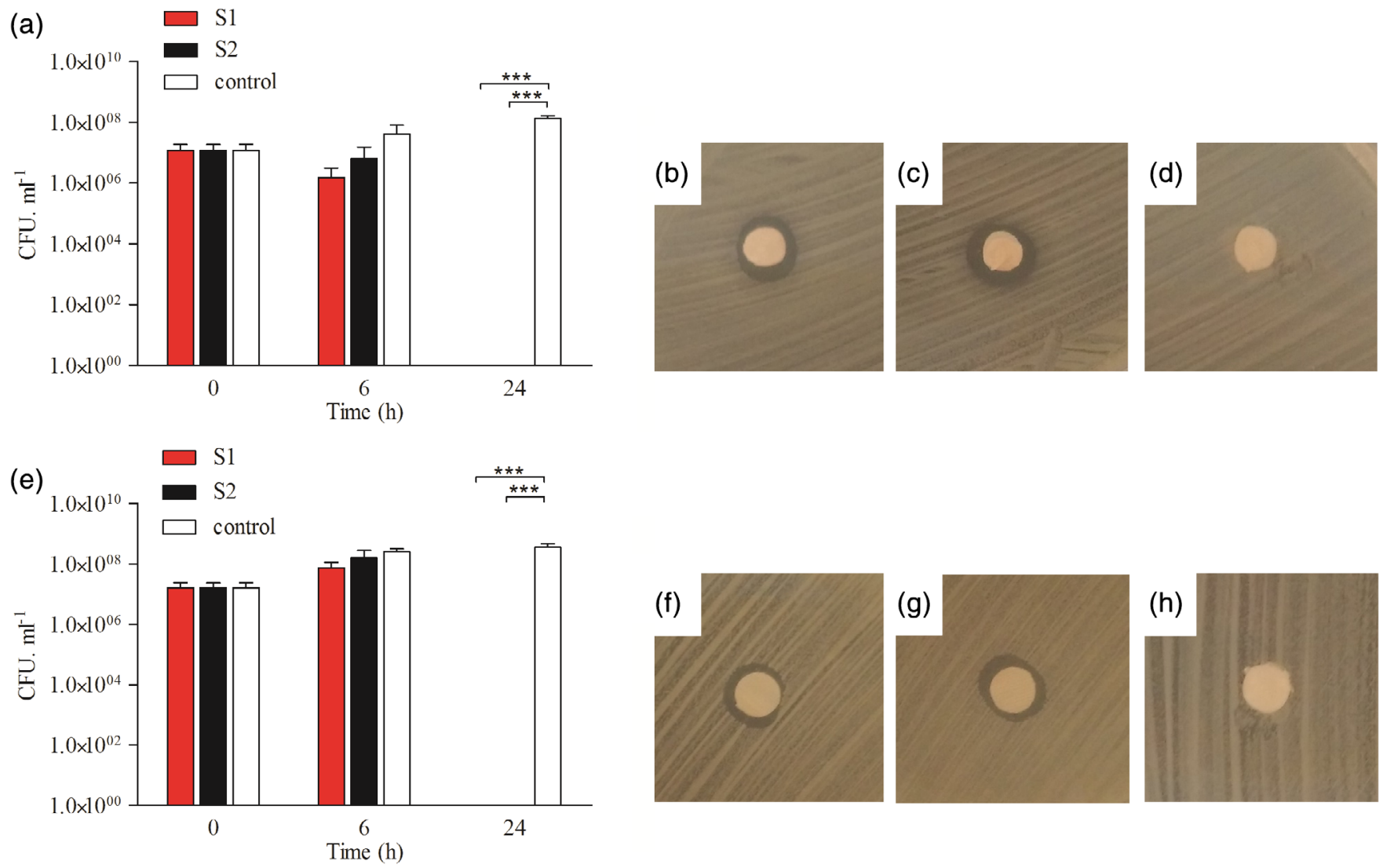

FIGURE 4 Antibacterial effect of S1 and S2 grafts. Plate counting of viable E. coli (a) and S. aureus (e) after incubation with S1, S2, and control grafts. Evaluation of the antibacterial potential of S1 and S2 grafts using the disk diffusion susceptibility test. Inhibition zone of (b) S1, (c) S2, and (d) control disks of E. coli and (f) S1, (g) S2, and (h) control disks for S. aureus after $24 \mathrm{hr}$ incubation at $37^{\circ} \mathrm{C}$. Data are expressed as mean \pm SD $(n=4)$ and $* * * p<.001$

The antibacterial effect of controlled NO delivery in this study is in agreement with earlier reports where NO-releasing significantly killed both S. aureus (Lee et al., 2016; Mihu et al., 2017; Seabra et al., 2010) and E. coli (Kabirian, Ditkowski, et al., 2019; Lee et al., 2016). The advantage of the antibacterial properties of the NO controlled release system is that it does not cause the development of bacterial resistance due to its multiple nonspecific bactericidal mechanisms (Backlund, Worley, \& Schoenfisch, 2016).

\section{6 | Effect of graft's extract on proliferation, morphology, and migration of ECs}

Following the SDVG implantation, the coverage of the luminal surface with ECs is required for healing and integration of grafts with the native tissue. Therefore, stimulation of ECs to migrate from the surrounding tissue and proliferate onto the newly implanted surface is crucial for the long-term clinical success of implantation.

Nitric oxide has different physiological properties such as stimulation of migration and proliferation of ECs when the release kinetic is in the physiological range (Li et al., 2018; Yang et al., 2015). As a proof of concept for physiological NO-release, the proliferation and migration of ECs in the presence of fabricated grafts were investigated.
Figure 5a shows the phase contrast images of HUVECs at the beginning of the experiment and after $24 \mathrm{hr}$ incubation with different extracts or culture medium. The initial location of the scratch wound is determined by dotted lines which represent the migration of ECs toward the cell-free zone. This cell-free zone is a model for the graft surface which should be covered with migrated ECs. The results (Figure 5b) illustrate that EC migration in presence of S1 and S2 extracts was significantly increased compared to both, the control extract and culture medium. This finding confirms the stimulating effect of NO on the migration rate of ECs which can promisingly increase the healing and integration of grafts. This faster endothelial coverage of grafts can also reduce the risk of bacterial colonization which consequently enhances the clinical success of the implant. A plausible explanation for this finding may be that the exogenous NO donor can upregulate the expression of cytoskeletal proteins known as VASP in ECs that are crucial for the elongation of actin filaments which subsequently enhance the migration (Segal et al., 2006). The results are in accordance with earlier studies that showed a significant increase in HUVEC proliferation and migration in the presence of exogenous NO donor supplementation (Kabirian, Milan, et al., 2019; Li et al., 2018; Yang et al., 2015).

The migration rate was not significantly different between S1 and S2 extracts which leads to the conclusion that both conditions are 
(a)

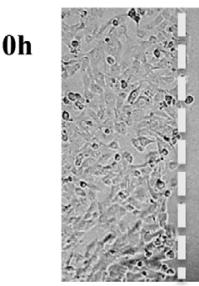

S1

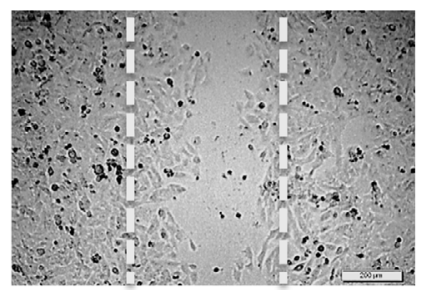

S2
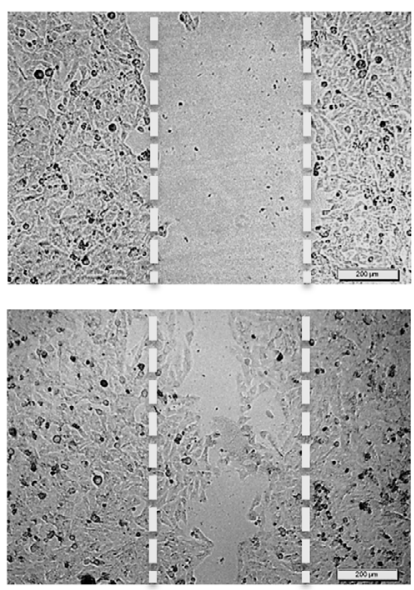

Control
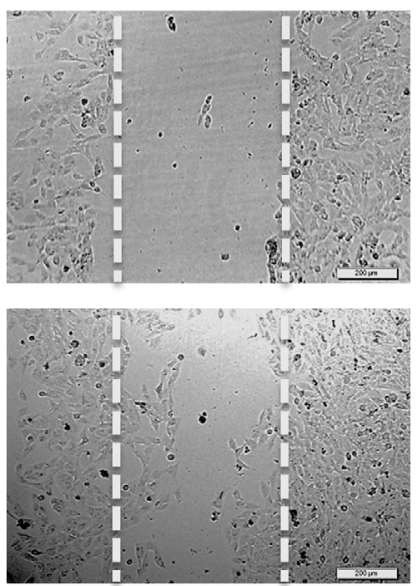

Culture medium
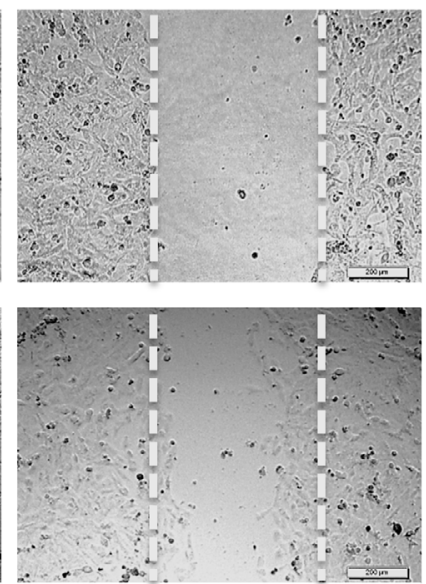

(b)

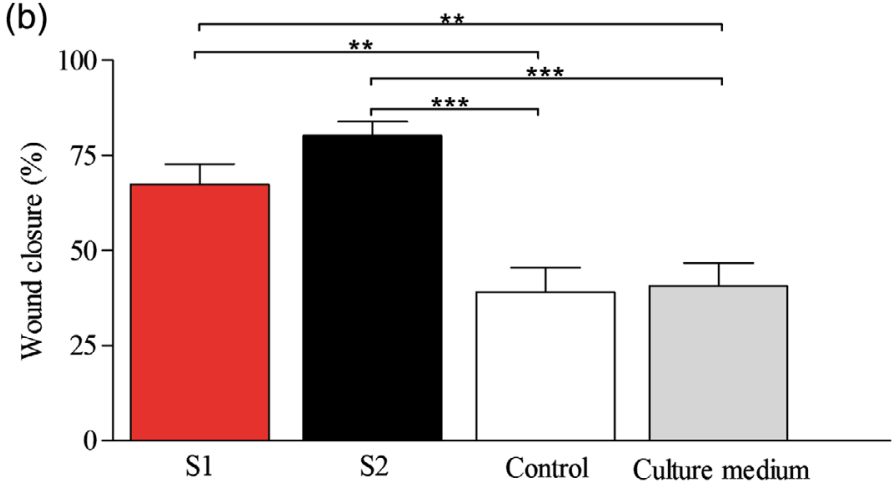

FIGURE 5 Assessment of the migration of HUVECs by scratch wound assay in vitro. (a) The monolayer of HUVECs which scratched with a $100-\mu l$ pipette tip (upper panel) and the same scratches after $24 \mathrm{hr}$ incubation with different extracts or culture medium (lower panel). Scale bar: $200 \mu \mathrm{m}$. (b) The percentage of wound closure calculated by comparison of the distance of wound edges at 0 and $24 \mathrm{hr}(n=3)$. HUVEC, Human umbilical vein endothelial cell

promising candidates to be used for clinical application. Interestingly, the migration rate of ECs in the presence of control extract or culture medium was not significantly different.

The proliferation of ECs in the presence of fabricated graft's extracts was also investigated. Figure 6 shows the phase contrast images of ECs after $24 \mathrm{hr}$ incubation with different extracts. ECs in all conditions reveal the elongated morphology showing the appropriate environment for cell growth. The cells proliferated in all conditions, however growing more confluent in the presence of NO-releasing extracts. It can thus be concluded that not only the fabricated grafts without NO-releasing are biocompatible without inducing ECs damage, but also the NO-releasing from both S1 and S2 can improve the proliferation of ECs and, subsequently, a higher endothelium coverage of S1 and S2 grafts can be expected in vivo.

\section{7 | Cytocompatibility assessment}

The evaluation of cytocompatibility of the newly fabricated biomaterials or chemical agents is essential since they can influence cell viability and metabolism. Because the ECs should cover SDVGs and be in direct contact with the surface, the viability of HUVECs was examined using the MTT assay. The results (Figure 7) confirm that the SDVGs support the proliferation of ECs. Moreover, in presence of extracts from S1 and S2 the proliferation of ECs significantly increased compared to the control condition while the viability of S1 or S2 incubated ECs was not significantly different. It can be concluded that the prepared SDVGs are noncytotoxic both in short-term (24 hr) and longer-term (72 hr) periods. The findings are in agreement with previous reports of zero toxicity and higher proliferation rate of ECs after NO treatment (Goudie, Pant, \& Handa, 2017; Kabirian, Milan, et al., 2019; Yang et al., 2015).

\section{4 | CONCLUSION}

In the current study, a modified SNAP impregnation method was introduced and incorporated in the production of 3D-printed SDVGs. This approach can shorten the preparation process while it is simple and cost-effective. The results demonstrate a controlled NO-release in the physiological range for a 15 days period. The created SDVGs showed the significant enhancement of ECs migration and proliferation while significantly killing and inhibiting bacterial growth. Considering these promising findings, SNAP-impregnated 3D-printed SDVGs can be used for fabrication of the next generation of antibacterial SDVGs with improved healing properties. 
S1
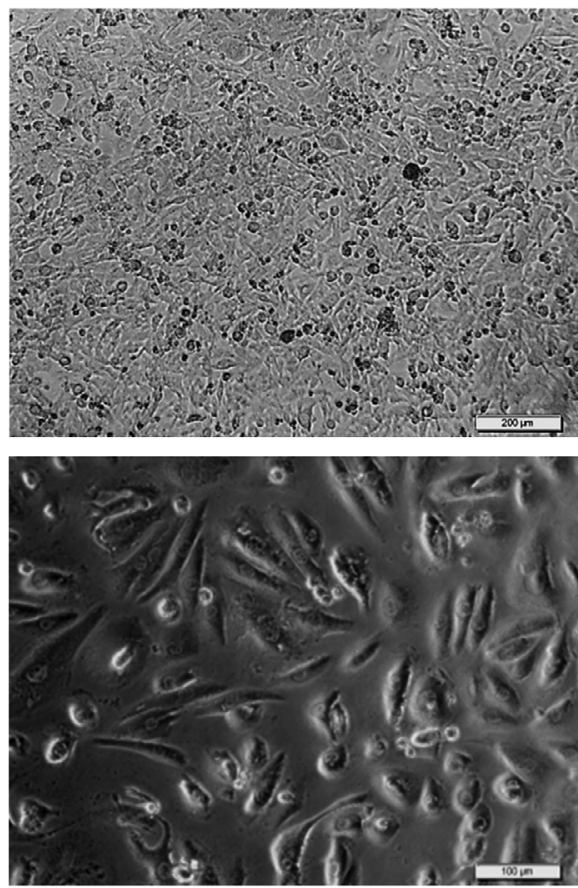

S2
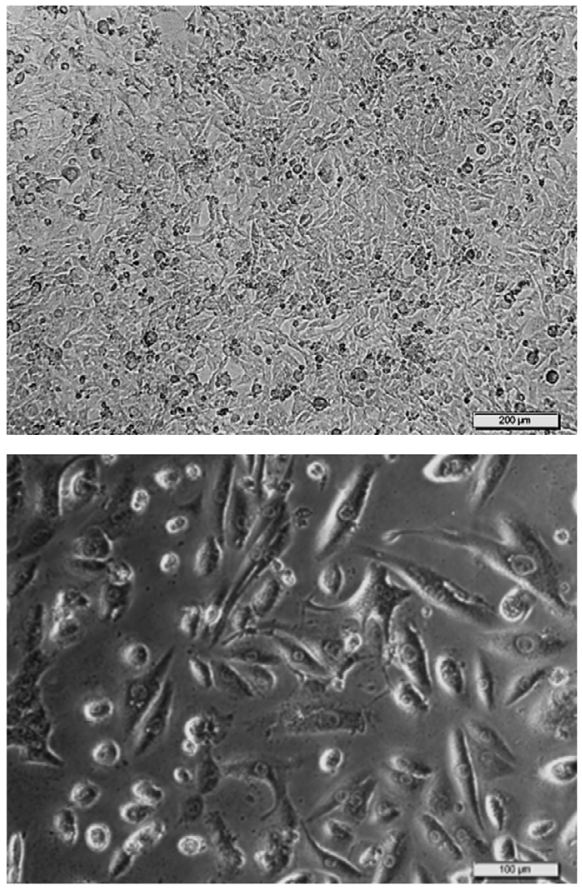

Control
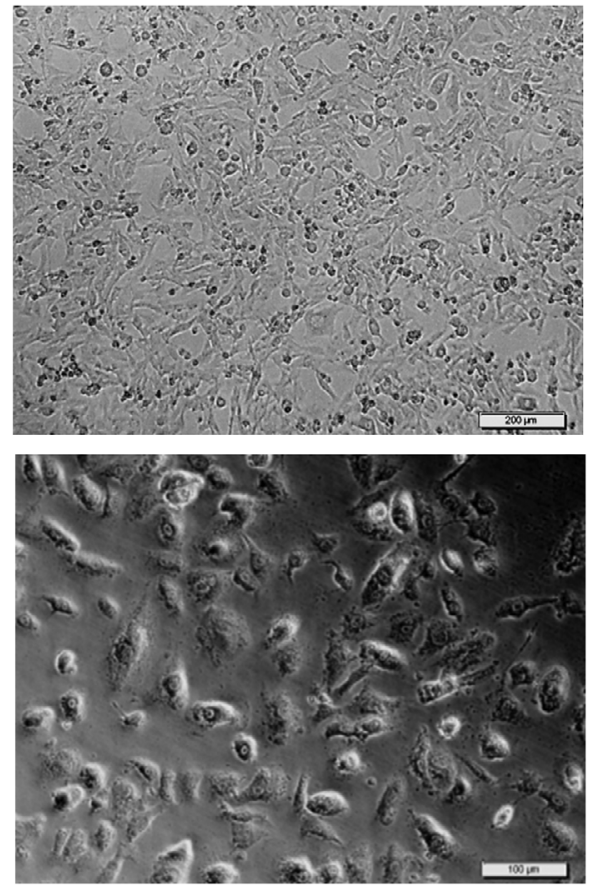

FIGURE 6 Proliferation and morphology of HUVECs after 24 hr incubation with S1, S2, or control graft's extracts. Images obtained by inverted microscopy. Scale bar: Upper panel: $200 \mu \mathrm{m}$ and lower panel: $100 \mu \mathrm{m}$. HUVEC, Human umbilical vein endothelial cell

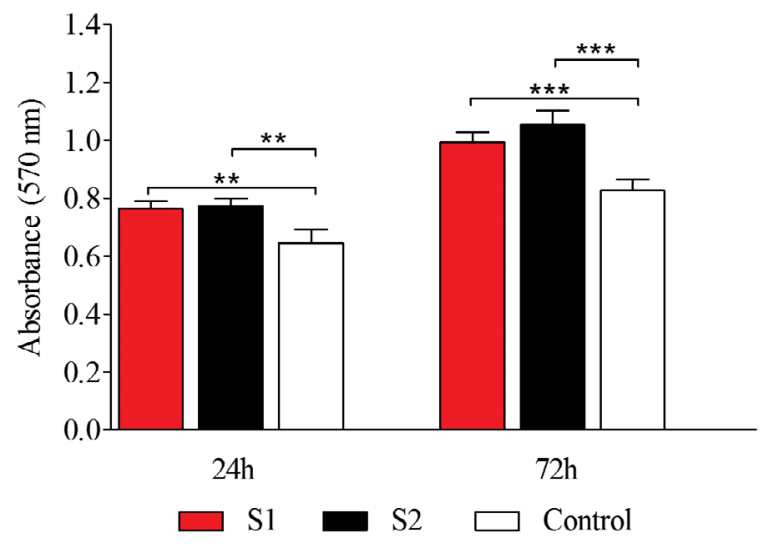

FIGURE 7 Cytocompatibility assessment of S1, S2, and control grafts. The HUVECs viability measured by MTT assay after 24 and $72 \mathrm{hr}$ incubation with different extracts. HUVEC, Human umbilical vein endothelial cell

\section{ORCID}

Fatemeh Kabirian (D) https://orcid.org/0000-0002-1626-8146 Masoud Mozafari (D) https://orcid.org/0000-0002-0232-352X

\section{REFERENCES}

Backlund, C. J., Worley, B. V., \& Schoenfisch, M. H. (2016). Anti-biofilm action of nitric oxide-releasing alkyl-modified poly (amidoamine) dendrimers against Streptococcus mutans. Acta Biomaterialia, 29, 198-205. https://doi.org/10.1016/j.actbio.2015.10.021

Brisbois, E. J., Major, T. C., Goudie, M. J., Bartlett, R. H., Meyerhoff, M. E., \& Handa, H. (2016). Improved hemocompatibility of silicone rubber extracorporeal tubing via solvent swelling-impregnation of S-nitroso- $\mathrm{N}$ acetylpenicillamine (SNAP) and evaluation in rabbit thrombogenicity model. Acta Biomaterialia, 37, 111-119. https://doi.org/10.1016/j. actbio.2016.04.025

Carpenter, A. W., \& Schoenfisch, M. H. (2012). Nitric oxide release: Part II. Therapeutic applications. Chemical Society Reviews, 41, 3742-3752. https://doi.org/10.1039/c2cs15273h

Chipinda, I., \& Simoyi, R. H. (2006). Formation and stability of a nitric oxide donor: S-nitroso- $\mathrm{N}$-acetylpenicillamine. The Journal of Physical Chemistry B, 110, 5052-5061. https://doi.org/10.1021/jp0531107

Colletta, A., Wu, J., Wo, Y., Kappler, M., Chen, H., Xi, C., \& Meyerhoff, M. E. (2015). S-Nitroso-N-acetylpenicillamine (SNAP) impregnated silicone foley catheters: A potential biomaterial/device to prevent catheterassociated urinary tract infections. ACS Biomaterials Science \& Engineering, 1, 416-424. https://doi.org/10.1021/acsbiomaterials.5b00032

Fang, F. C. (1997). Perspectives series: Host/pathogen interactions. Mechanisms of nitric oxide-related antimicrobial activity. The Journal of Clinical Investigation, 99, 2818-2825. https://doi.org/10.1172/JCI119473

Gao, J., Wang, Y., Chen, S., Tang, D., Jiang, L., Kong, D., \& Wang, S. (2017). Electrospun poly- $\varepsilon$-caprolactone scaffold modified with catalytic nitric oxide generation and heparin for small-diameter vascular graft. RSC Advances, 7, 18775-18784. https://doi.org/10.1039/C7RA02086D

García-Robledo, E., Corzo, A., \& Papaspyrou, S. (2014). A fast and direct spectrophotometric method for the sequential determination of nitrate and nitrite at low concentrations in small volumes. Marine Chemistry, 162, 30-36. https://doi.org/10.1016/j.marchem.2014.03.002

Goudie, M. J., Brainard, B. M., Schmiedt, C. W., \& Handa, H. (2017). Characterization and in vivo performance of nitric oxide-releasing extracorporeal circuits in a feline model of thrombogenicity. Journal of Biomedical Materials Research Part A, 105, 539-546. https://doi.org/ 10.1002/jbm.a.35932

Goudie, M. J., Pant, J., \& Handa, H. (2017). Liquid-infused nitric oxidereleasing (LINORel) silicone for decreased fouling, thrombosis, and infection of medical devices. Scientific Reports, 7, 13623. https://doi. org/10.1038/s41598-017-14012-9 
Kabirian, F., Amoabediny, G., Haghighipour, N., Salehi-Nik, N., \& ZandiehDoulabi, B. (2015). Nitric oxide secretion by endothelial cells in response to fluid shear stress, aspirin, and temperature. Journal of Biomedical Materials Research Part A, 103, 1231-1237. https://doi.org/ 10.1002/jbm.a.35233

Kabirian, F., Ditkowski, B., Zamanian, A., Heying, R., \& Mozafari, M. (2018). An innovative approach towards 3D-printed scaffolds for the next generation of tissue-engineered vascular grafts. Materials Today: Proceedings, 5, 15586-15594. https://doi.org/10.1016/j.matpr.2018. 04.167

Kabirian, F., Ditkowski, B., Zamanian, A., Hoylaerts, M. F., Mozafari, M., \& Heying, R. (2019). Controlled NO-release from 3D-printed smalldiameter vascular grafts prevents platelet activation and bacterial infectivity. ACS Biomaterials Science \& Engineering, 5(5), 2284-2296. https://doi.org/10.1021/acsbiomaterials.9b00220

Kabirian, F., Milan, P. B., Zamanian, A., Heying, R., \& Mozafari, M. (2019). Nitric oxide-releasing vascular grafts: $A$ therapeutic strategy to promote angiogenic activity and endothelium regeneration. Acta Biomaterialia, 92, 82-91. https://doi.org/10.1016/j.actbio.2019.05.002

Kabirian, F., \& Mozafari, M. (2019). Decellularized ECM-derived bioinks: Prospects for the future. Methods. https://www.sciencedirect. com/science/article/pii/S1046202318304377

Lee, W. H., Ren, H., Wu, J., Novak, O., Brown, R. B., Xi, C., \& Meyerhoff, M. E. (2016). Electrochemically modulated nitric oxide release from flexible silicone rubber patch: Antimicrobial activity for potential wound healing applications. ACS Biomaterials Science \& Engineering, 2, 1432-1435. https://doi.org/10.1021/acsbiomaterials. $6 \mathrm{~b} 00360$

Li, X., Qiu, H., Gao, P., Yang, Y., Yang, Z., \& Huang, N. (2018). Synergetic coordination and catecholamine chemistry for catalytic generation of nitric oxide on vascular stents. NPG Asia Materials, 1, 482-496. https://doi.org/10.1038/s41427-018-0052-3

Mihu, M. R., Cabral, V., Pattabhi, R., Tar, M. T., Davies, K. P., Friedman, A. J., ... Nosanchuk, J. D. (2017). Sustained nitric oxidereleasing nanoparticles interfere with methicillin-resistant Staphylococcus aureus adhesion and biofilm formation in a rat central venous catheter model. Antimicrobial Agents and Chemotherapy, 61, e02020-e02016. https://doi.org/10.1128/AAC.02020-16

Miranda, K. M., Espey, M. G., \& Wink, D. A. (2001). A rapid, simple spectrophotometric method for simultaneous detection of nitrate and nitrite. Nitric Oxide, 5, 62-71. https://doi.org/10.1006/niox.2000.0319

Pant, J., Gao, J., Goudie, M. J., Hopkins, S. P., Locklin, J., \& Handa, H. (2017). A multi-defense strategy: Enhancing bactericidal activity of a medical grade polymer with a nitric oxide donor and surfaceimmobilized quaternary ammonium compound. Acta Biomaterialia, 58, 421-431. https://doi.org/10.1016/j.actbio.2017.05.061

Pant, J., Goudie, M. J., Chaji, S. M., Johnson, B. W., \& Handa, H. (2018). Nitric oxide releasing vascular catheters for eradicating bacterial infection. Journal of Biomedical Materials Research Part B: Applied Biomaterials, 106, 2849-2857. https://doi.org/10.1002/jbm.b.34065

Pashneh-Tala, S., MacNeil, S., \& Claeyssens, F. (2015). The tissue-engineered vascular graft-Past, present, and future. Tissue Engineering Part $B$ : Reviews, 22, 68-100. https://doi.org/10.1089/ten.teb.2015.0100

Radakovic, D., Reboredo, J., Helm, M., Weigel, T., Schürlein, S., Kupczyk, E., ... Hansmann, J. (2017). A multilayered electrospun graft as vascular access for hemodialysis. PLoS ONE, 12, e0185916.

Rao, B. R., Kotcherlakota, R., Nethi, S. K., Puvvada, N., Sreedhar, B., Chaudhuri, A., \& Patra, C. R. (2018). Ag2 [Fe (CN) 5NO] nanoparticles exhibit antibacterial activity and wound healing properties. ACS
Biomaterials Science \& Engineering, 4, 3434-3449. https://doi.org/10. 1021/acsbiomaterials.8b00759

Riccio, D. A., \& Schoenfisch, M. H. (2012). Nitric oxide release: Part I. Macromolecular scaffolds. Chemical Society Reviews, 41, 3731-3741. https://doi.org/10.1039/c2cs15272j

Seabra, A. B., Martins, D., Simões, M. M., Da Silva, R., Brocchi, M., \& De Oliveira, M. G. (2010). Antibacterial nitric oxide-releasing polyester for the coating of blood-contacting artificial materials. Artificial Organs, 34, E204-E214. https://doi.org/10.1111/j.1525-1594

Segal, M. S., Shah, R., Afzal, A., Perrault, C. M., Chang, K., Schuler, A., ... Tran-Son-Tay, R. (2006). Nitric oxide cytoskeletal-induced alterations reverse the endothelial progenitor cell migratory defect associated with diabetes. Diabetes, 55, 102-109. https://doi.org/10.2337/ diabetes.55.01.06.db05-0803

Su, M., Huang, J., Liu, S., Xiao, Y., Qin, X., Liu, J., ... Luo, Z. (2016). The antiangiogenic effect and novel mechanisms of action of Combretastatin A-4. Scientific Reports, 6, 28139. https://doi.org/10.1038/srep28139

Thi, T. T. H., Lee, Y., Le Thi, P., \& Park, K. D. (2018). Nitric oxide-releasing injectable hydrogels with high antibacterial activity through in situ formation of peroxynitrite. Acta Biomaterialia, 67, 66-78. https://doi.org/ 10.1016/j.actbio.2017.12.005

Touri, M., Kabirian, F., Saadati, M., Ramakrishna, S., \& Mozafari, M. (2019). Additive manufacturing of biomaterials-The evolution of rapid prototyping. Advanced Engineering Materials, 21, 1800511. https://doi. org/10.1002/adem.201800511

Vermote, A., \& Van Calenbergh, S. (2017). Small-molecule potentiators for conventional antibiotics against Staphylococcus aureus. ACS infectious diseases, 3, 780-796. https://doi.org/10.1021/acsinfecdis.7b00084

Wo, Y., Brisbois, E. J., Wu, J., Li, Z., Major, T. C., Mohammed, A., ... Xi, C. (2017). Reduction of thrombosis and bacterial infection via controlled nitric oxide (NO) release from S-nitroso-N-acetylpenicillamine (SNAP) impregnated CarboSil intravascular catheters. ACS Biomaterials Science \& Engineering, 3, 349-359. https://doi.org/10.1021/acsbiomaterials.6b00622

Wo, Y., Li, Z., Brisbois, E. J., Colletta, A., Wu, J., Major, T. C., ... Meyerhoff, M. E. (2015). Origin of long-term storage stability and nitric oxide release behavior of CarboSil polymer doped with S-nitroso$\mathrm{N}$-acetyl-D-penicillamine. ACS Applied Materials \& Interfaces, 7, 22218-22227. https://doi.org/10.1021/acsami.5b07501

Yang, Z., Yang, Y., Xiong, K., Li, X., Qi, P., Tu, Q., ... Huang, N. (2015). Nitric oxide producing coating mimicking endothelium function for multifunctional vascular stents. Biomaterials, 63, 80-92. https://doi. org/10.1016/j.biomaterials.2015.06.016

Zamani, M., Khafaji, M., Naji, M., Vossoughi, M., Alemzadeh, I., \& Haghighipour, N. (2017). A biomimetic heparinized composite silkbased vascular scaffold with sustained antithrombogenicity. Scientific Reports, 7, 4455. https://doi.org/10.1038/s41598-018-32859-4

Zilberman, M., \& Elsner, J. J. (2008). Antibiotic-eluting medical devices for various applications. Journal of Controlled Release, 130, 202-215. https://doi.org/10.1016/j.jconrel.2008.05.020

How to cite this article: Kabirian F, Brouki Milan P, Zamanian A, Heying R, Mozafari M. Additively manufactured small-diameter vascular grafts with improved tissue healing using a novel SNAP impregnation method. J Biomed Mater Res. 2019;1-10. https://doi.org/10.1002/jbm.b.34481 